\section{Domos Modulares Interconectados de Progresión Continua (Domos Amopa)}

Francisco Martínez Cendra ${ }^{(1)}$

Resumen: Las cúpulas geodésicas se vienen diseñando en diversos lugares partiendo principalmente de figuras poliédricas tales como el Icosaedro. Esto supone edificaciones de planta circular que por lo general deben actuar de modo independiente ya que la geometría propia de cada elemento no permite la unión de domos, a menos que se fuerce la geometría básica para lograr acoplar estas edificaciones a modo de pompas de jabón, donde en cuyo caso habrá que recortar diversos elementos a fin de lograr esta unión.

Esto representa un problema estructural que hay que solucionar mediante elementos ajenos a la geometría básica del domo y que de algún modo solucionen el trauma de haber seccionado la estructura en diversas partes.

El objetivo del presente artículo es estudiar una forma de diseño alternativa que permita la unión de domos sin alterar su propia geometría básica.

Palabras clave: Arquitectura orgánica - Unión de domos - Cúpulas geodésicas interconectadas

[Resúmenes en inglés y portugués en las páginas 129-130]

(1) Francisco Martínez Cendra. Maestro en Ciencias con mención en Arquitectura por la Universidad Nacional de Ingeniería. Docente de la Universidad Peruana de Ciencias Aplicadas UPC, Lima, Perú. Estudió arquitectura en la Universidad Ricardo Palma, realizó una maestría en la Universidad Nacional de Ingeniería y luego realizó un posgrado en la universidad Politécnica de Catalunya, España. En el año 2003 publica su primer libro titulado "Hacia Una Arquitectura Fractal", posteriormente en el año 2014 publica su segundo libro titulado “De Cúpulas Geodésicas, Fractales, Tensegritys Y Algo Más”, ambas publicaciones a cargo de la facultad de Ingeniería y Arquitectura de la Universidad San Martín de Porres donde se inició como catedrático a tiempo parcial en el año 2000. Actualmente es docente Investigador a tiempo completo en la Universidad Científica del Sur. Actualmente, ha terminado su tercer libro titulado "Arquitectura Fractales y Geometría” el cual se encuentra en etapa de revisión. fmcendra@gmail.com. (D) ORCID Nº000-0001-7979-0372 


\section{Domos Poliédricos}

Por definición los domos, también conocidos como cúpulas geodésicas, son unas estructuras tridimensionales basada en un poliedro que permanece inscrito en una esfera $y$, aunque por lo general estas estructuras se relacionan con la geometría del icosaedro, también se pueden generar domos a partir de los otros cuatro poliedros regulares y de forma general por cualquier otro poliedro que pueda inscribirse en una esfera. Es más, inclusive se pueden hacer domos al proyectar una forma poligonal a una esfera.

Por lo tanto, la forma tradicional de diseñar un domo consiste en inscribir uno de estos cinco poliedros en una esfera y empezar a proyectar los diferentes puntos obtenidos por la frecuencia o subdivisión de cada una de las caras del poliedro a algunos de los infinitos círculos que forman la esfera (Ver Figura 1).

De esta manera tendremos domos poliédricos basados en cada uno de estos cinco poliedros regulares.

\section{Unión de Domos}

La arquitectura mostrada en Eden Project, diseñado por Grimshaw Architects es una excelente muestra arquitectónica basada en la unión de domos (Ver Figura 2). Esta configuración geométrica permite cubrir grandes luces a fin de obtener espacios libres de apoyos intermedios, sin embargo y tal como se puede apreciar en la figura anterior, para lograr la unión entre cúpulas geodésicas ha sido necesario recortar las barras estructurales y añadir una cercha con forma de arco que no es otra cosa que un elemento ajeno a la geometría del domo. Si no fuera por esta cercha los elementos seccionados no tendrían cómo unirse unos con otros de forma segura.

Como se puede apreciar en la Figura 3, la única manera de lograr una unión entre dos cúpulas geodésicas es seccionando algunas barras a fin de lograr la unión entre los domos. La disposición geométrica de estos elementos no facilita la unión entre ellos debido a que en la propia geometría de los domos no existen elementos que, de algún modo, puedan encajar unos con otros a fin de poder acoplarse sin necesidad de cortarlos y de añadir un elemento adicional tal como una cercha que solucione el trauma estructural generado. El objetivo de este trabajo es desarrollar un domo geodésico que pueda unirse con otro de una manera similar a la forma que emplea la propia naturaleza para unir, por ejemplo, las pompas de jabón. Si observamos con detenimiento la Figura 4 veremos que las pompas de jabón se unen mediante un plano perpendicular a la base de apoyo, esto debería darnos pie a investigar un tipo de estructura esférica en la cual podamos aplicar este concepto.

La naturaleza es sabia y ella siempre buscará la forma más rápida y eficiente para hacer las cosas, es por ello que grandes pensadores y arquitectos como Frei Otto afirmaba que toda forma que se pueda obtener con pompas de jabón puede aplicarse a una estructura neumática (Otto, 1973). Del mismo modo Antoni Gaudí, desde su temprana edad, le inspiraron las formas naturales que luego le ayudarían a realizar sus célebres obras, recordemos las maquetas funiculares que mantenían su forma por acción de la gravedad 

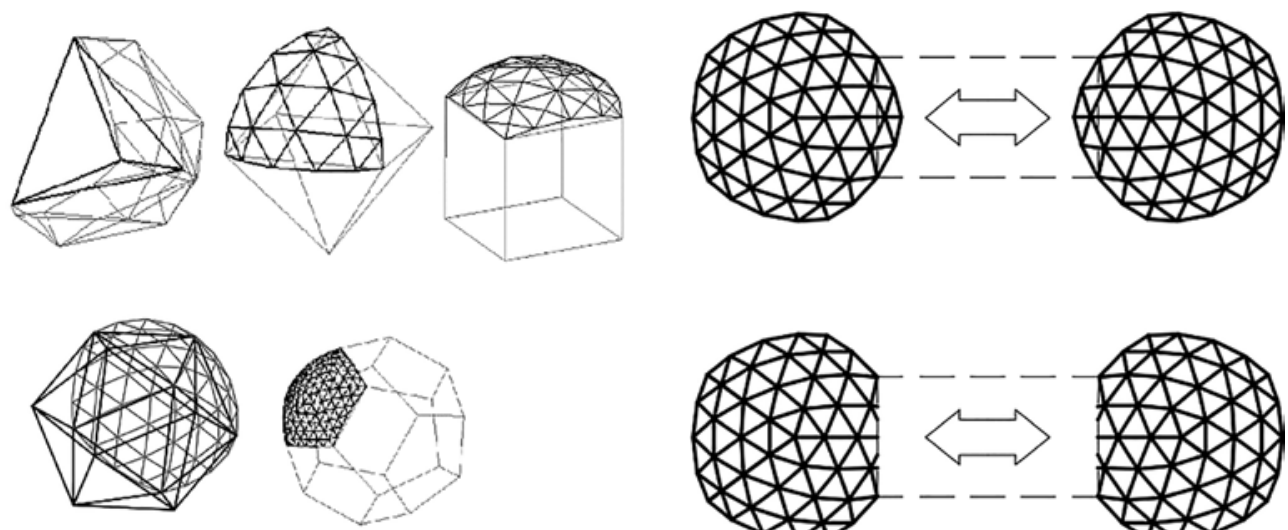

1

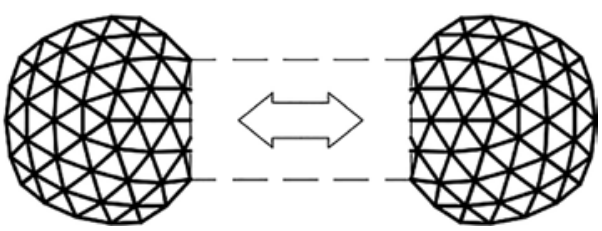

3
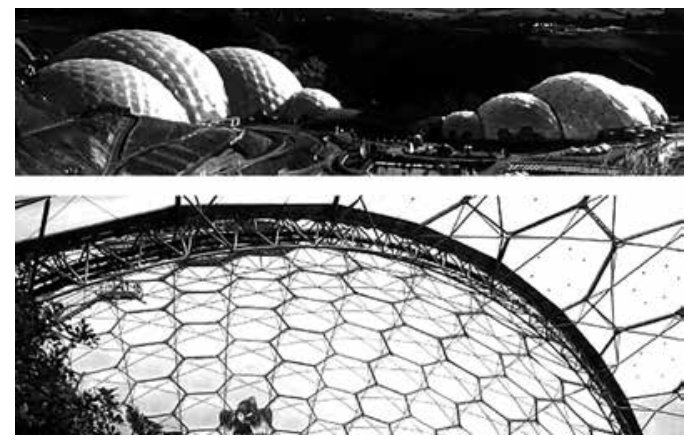

2

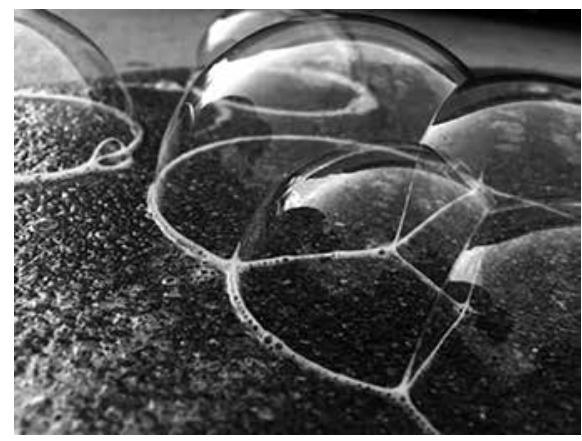

4

Figura 1. Formación de secciones esféricas a partir de un tetraedro, un octaedro, un hexaedro, un icosaedro y un dodecaedro (Gráfico realizado por el autor). Figura 2. Unión de domos geodésicos forzando la geometría básica (Fuente de las fotos: https://www.transitionculture.org/2006/06/14/5-great-things-about-the-eden-project-3-thebiomes/). Figura 3. Unión forzada de domos (Gráfico realizado por el autor). Figura 4. Unión de pompas de jabón (Fuente: Internet). 
y que sirvieron de modelo para que este genio catalán de la arquitectura hiciera bóvedas tan características como las de la Sagrada Familia. Al respecto de Gaudí, Joan Bassegoda nos comenta:

Tuvo la gran habilidad de saber ver a su alrededor toda la maravilla de la creación. Entendió como nadie el valor de la luz, del color y del brillo, amó los sonidos, percibió los olores y, en fin, dejó en perpetua receptividad sus cinco sentidos para mejor conocer y amar, con sentimiento claramente franciscano, las maravillas de la creación.

Estas espléndidas lecciones de arte, arquitectura y técnica que la botánica, la geología o la zoología le ofrecían fueron la base de su pensamiento de artista creador (Bassegoda, 1985, p. 45).

\section{Domos Amopa}

Tal como mencionamos más arriba, la solución a este problema reside en diseñar una estructura, basada en arcos paralelos y perpendiculares a la base sobre la cual se asienta el domo de la misma forma que la naturaleza une las pompas de jabón, de tal suerte que se logrará una perfecta unión entre las cúpulas para, de esta manera, generar conjuntos modulares que puedan crecer de acuerdo a las necesidades del usuario. Este tipo de domos los denominaremos Domos Amopa (Arcos Modulares Paralelos) (Ver Figura 5).

Iniciamos el diseño basado en una trama geométrica que oficiará como ente organizador entre los distintos elementos que conformarán la estructura y sobre la cual trazaremos los elementos que nos permitirán diseñar este domo. En la Figura 5 vemos como se ha trazado sobre la trama, un cuadrado que a su vez contiene tres cuadrados de la trama por lado, esto nos indica en este ejemplo, que el domo será de frecuencia ${ }^{1}$ tres, si el cuadrado contuviera cuatro o cinco cuadrados de la trama entonces sería de frecuencia cuatro o cinco y así sucesivamente.

Luego trazamos arcos perpendiculares a la base sobre las aristas de este cuadrado que llamaremos cuadrado matriz, luego sobre las líneas paralelas a las aristas de este cuadrado matriz y finalmente sobre las respectivas diagonales (Ver Figura 5), la intersección de estos arcos determinará los puntos de unión de las barras que determinarán el domo. Como se aprecia, los arcos cambiarán de altura según el arco que pasa por la diagonal del cuadrado matriz, de esta manera obtendremos el casquete de una esfera que será la matriz del domo. Sin embargo, hay que tener en cuenta lo siguiente, si empleamos medio círculo (en elevación) como base para trazar los respectivos arcos perpendiculares a la base, ocurrirá que a medida que los planos paralelos se acercan a un extremo, aumenta de forma exponencial la distancia de los puntos de corte en la esfera, tal como podemos apreciar en la Figura 6 donde vemos que las distancias entre los segmentos - del punto A al punto E- son de medidas similares a los demás segmentos no así el último segmento EF. Esto hace que las barras del domo pierdan proporcionalidad haciendo que las barras ubicadas en los extremos cerca a la base resulten excesivamente grandes en comparación con las barras de 

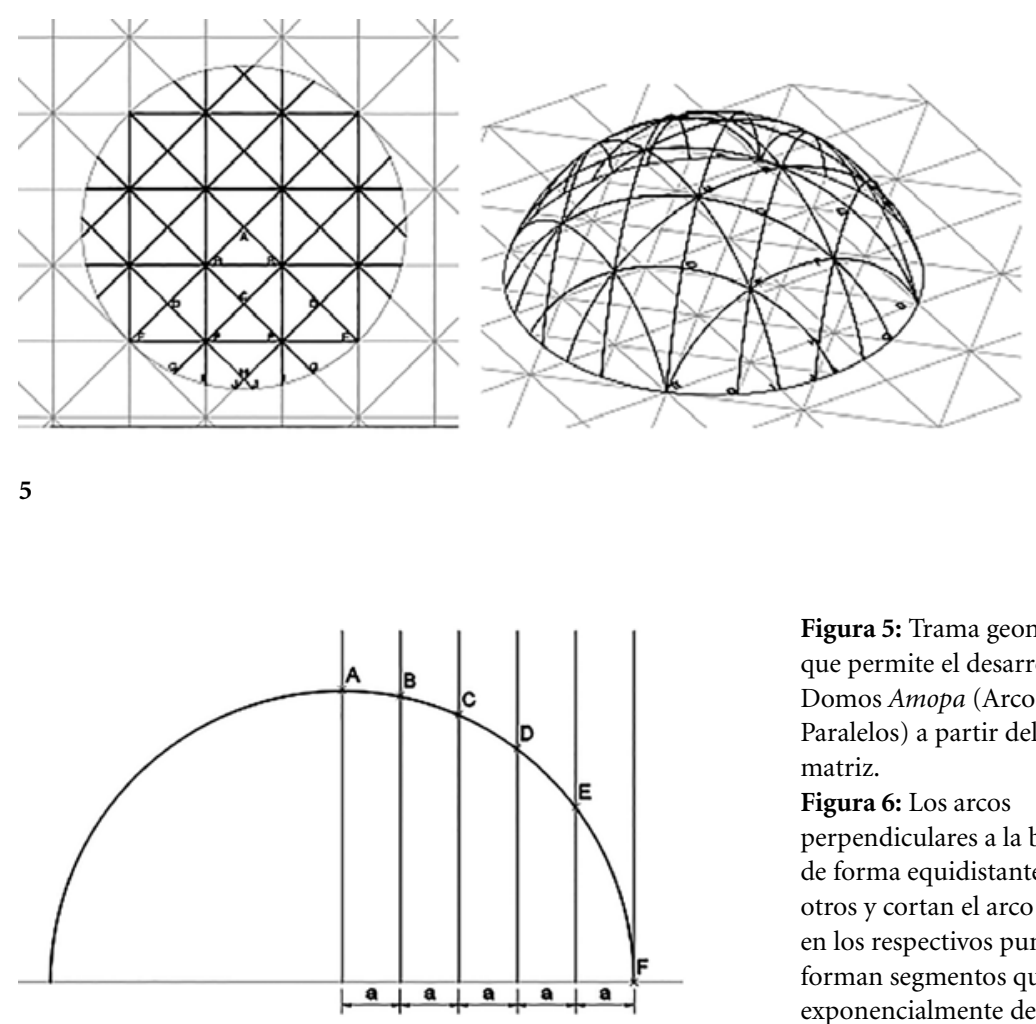

Figura 5: Trama geométrica que permite el desarrollo de los Domos Amopa (Arcos Modulares Paralelos) a partir del cuadrado matriz.

Figura 6: Los arcos

perpendiculares a la base se ubican de forma equidistante unos de otros y cortan el arco del centro en los respectivos puntos que forman segmentos que aumentan exponencialmente de longitud.

6

la parte más alta, lo que genera un problema de diseño, pues el domo que mantenía una forma proporcional en la parte alta y media, pierde esta característica en la zona inferior (Ver Figura 6).

En los domos tradicionales -icosaédricos- esto no ocurre, las barras mantienen una proporcionalidad tal que inclusive muchas personas piensan que son iguales, al extremo que afirman que un domo está compuesto por triángulos equiláteros, lo cual es totalmente falso.

Se pueden ensayar diversas soluciones al respecto, una de ellas es evitar que el centro de radio del domo esté en el plano de la base tal como en los domos tradicionales, ubicándose por debajo de la superficie de apoyo, por lo tanto, el Domo Amopa no será nunca media esfera, será un casquete esférico. 
Existen varias soluciones al respecto, a continuación, ensayaremos una de ellas:

- Dividimos en 10 partes el radio de la base del círculo de la esfera que contendrá el domo de tal manera que la base del domo se situará por debajo a 3/10 de él en el punto $O$, por debajo del plano de referencia donde se ubica la trama cuadrada y se ubica el punto O', el cual es el centro de radio de la base en la cual se asienta el domo. Entonces, desde este punto $\mathrm{O}$ trazamos el arco maestro que será el referente para los demás arcos perpendiculares a la base y paralelos a este. Todos los demás arcos mostrados en la siguiente figura tendrán una altura máxima que pasará por la intersección de las líneas de la trama cuadrada y serán entre sí tanto rectos como oblicuos (Ver Figura 7).

De esta manera obtenemos los arcos perpendiculares como se aprecia en la Figura 7. El domo resultará de la intersección de estos arcos. Este domo resulta ser muy versátil pues permite unirse con otros de forma modular formando diversas composiciones. Se puede unir uno seguido de otro formando un conjunto de dos, tres o más domos. Se pueden unir, además de forma oblicua formando un ángulo de $90^{\circ}$ o de $45^{\circ}$ grados y sin necesidad de cortar ninguna barra lo que hace que no pierda su resistencia estructural y que, por supuesto, no sea necesario añadir una estructura adicional de refuerzo (Ver Figuras 8).

El domo Amopa presenta dos partes diferenciadas a saber, las barra comprendidas dentro del cuadrado matriz las cuales las llamaremos Barras de Crucería por la clara alusión a las cúpulas góticas y las barras comprendidas fuera del cuadrado matriz las cuales las llamaremos Barras de Contrafuerte. Esta diferenciación nos será de utilidad a la hora de diseñar a fin de poder ubicar con claridad los arcos de unión entre domos (Ver Tabla 1).

En las composiciones mostradas en las Figuras 9 y 10 se observan diferentes formas de unión de este domo el cual ha sido construido en frecuencia 3 y 2 respectivamente.

Para obtener la dimensión de cualquiera de las barras bastará con multiplicar el radio de la base sobre la cual se asentará el domo con la constante K relativa a cada barra según las tablas anexas al presente artículo, de esta manera se pueden realizar los modelos mostrados siguiendo el patrón de ensamble mostrado en la Figura 11. Un domo constará de cuatro triángulos esféricos, como los mostrados en la anterior figura, donde tenemos las piezas dibujadas de un domo de frecuencia dos a la izquierda y de frecuencia tres a la derecha en un plano en verdadera magnitud, las cuales al unirse formarán el respectivo domo. Pueden reconocerse las Barras de Crucería y las Barras de Contrafuerte.

$\mathrm{Al}$ igual que los domos tradicionales, estos se calculan sin considerar la forma de la barra estructural que se está empleando. El cálculo nos arrojará una dimensión exacta de la barra de forma alámbrica, es decir que luego habrá que considerar en el diseño el ancho y alto de la barra, así como la forma y medida del nudo de unión del domo. Por ejemplo, si realizamos un domo de la forma más simple que es aplastando los extremos de las barras para luego hacer una perforación por donde pasar un perno que unirá las barras, entonces la distancia que nos arroja el cálculo será del centro del orificio de un extremo al centro del orificio del extremo opuesto, es decir que luego habrá que sumarle a la barra unos centímetros adicionales para completar el nudo. 


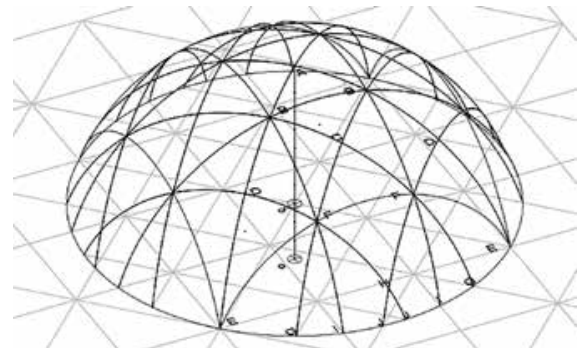

7
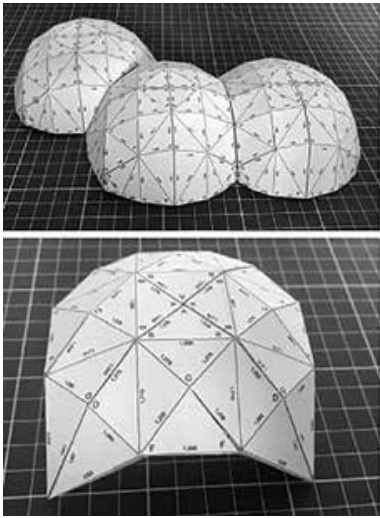

9
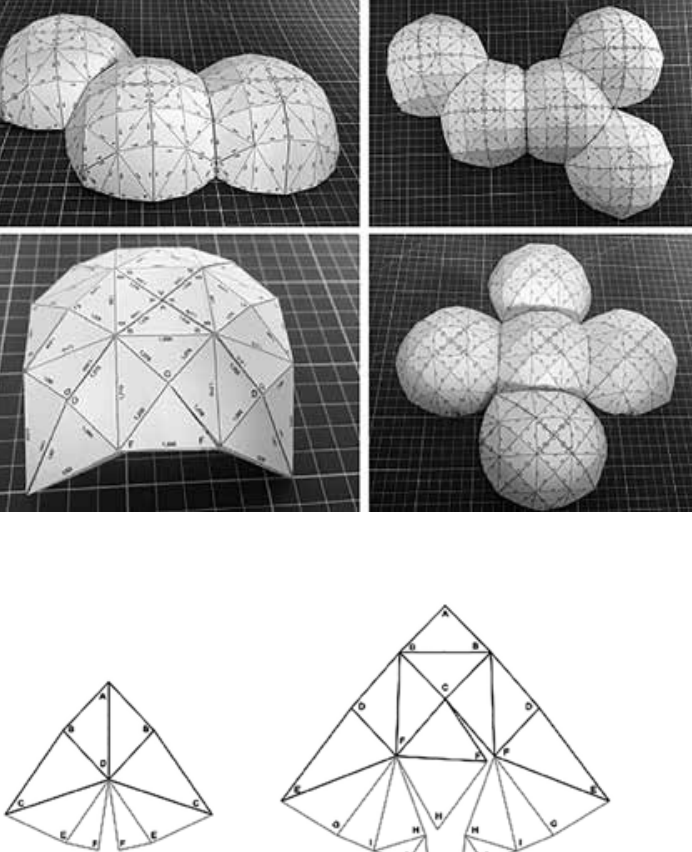

FRECUENCIA 2

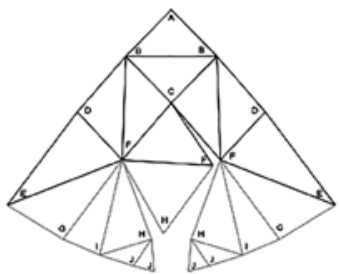

FRECUENCIA 3

8
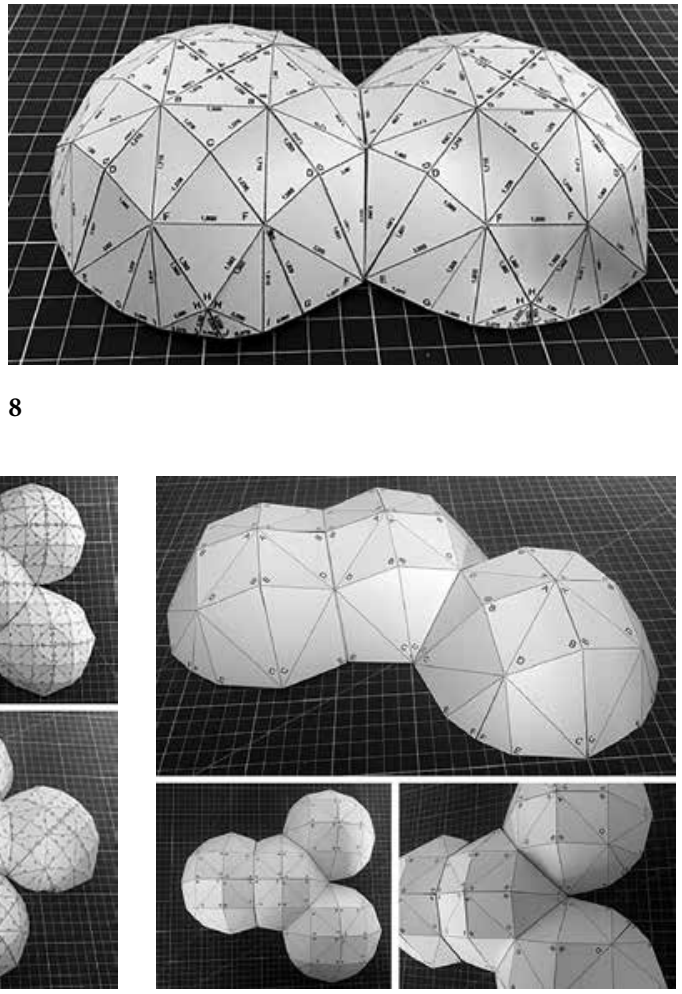

10

Figura 7: Arcos perpendiculares basados la trama cuadrada.

Figura 8: Maqueta de dos domos Amopa de frecuencia 3 unidos de forma axial.

Figura 9: Maqueta de domos Amopa de frecuencia 3 unidos de múltiples formas. Figura 10: Maqueta de domos Amopa de frecuencia 2 unidos de múltiples formas. Tabla 1. Domo Amopa de frecuencia dos. Figura 11: Patrón de ensamble para realizar un Domo Amopa de frecuencia dos y frecuencia tres. 

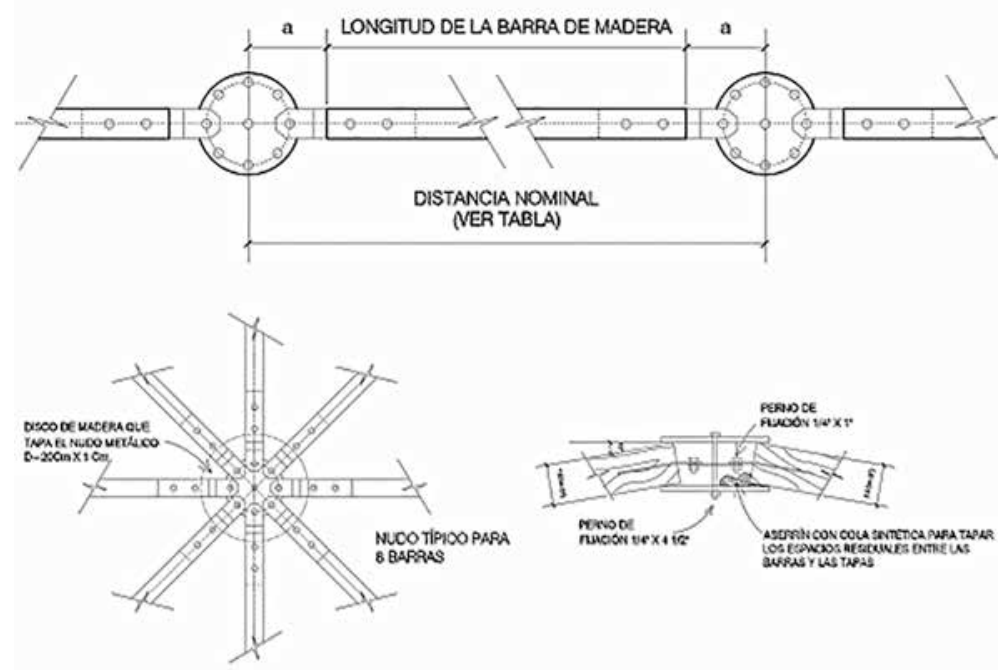

12

\begin{tabular}{|c|c|c|c|c|}
\hline & AMOPA F3 & Diedro & & \\
\hline & \multicolumn{2}{|c|}{ BARRAS DE CRUCERIA } & & \\
\hline 1 & $\mathrm{UAB}$ & 18.628860 & $\mathrm{KAB}=$ & 0.3379576 \\
\hline 2 & U BB & 26.095906 & $\mathrm{~K} \mathrm{BB}=$ & 0.4714142 \\
\hline 3 & U BC & 18.647783 & $\mathrm{~K} \mathrm{BC}=$ & 0.3382979 \\
\hline 4 & U BD & 21.065408 & $\mathrm{~K} \mathrm{BD}=$ & 0.3816908 \\
\hline 5 & U DF & 18.800960 & $K$ DF= & 0.3410519 \\
\hline 6 & U FF & 26.096241 & $\mathrm{KFF}=$ & 0.4714202 \\
\hline 7 & UFC & 21.471482 & $\mathrm{~K} \mathrm{FC}=$ & 0.3889631 \\
\hline 8 & UDE & 33.608038 & $\mathrm{~K} \mathrm{DE}=$ & 0.6036563 \\
\hline 9 & $\mathrm{UEF}$ & 35.631938 & $\mathrm{KEF}=$ & 0.6388646 \\
\hline \multirow{2}{*}{10} & U BF & 29.828244 & $\mathrm{~K} \mathrm{BF}=$ & 0.5374064 \\
\hline & \multicolumn{2}{|c|}{ BARRAS DE CONTRAFUERTE } & & - \\
\hline 11 & UEG & 18.650559 & $K$ EG= & 0.3383478 \\
\hline 12 & U GF & 28.382850 & K GF= & 0.5119139 \\
\hline 13 & $\mathrm{U}$ GI & 11.405318 & $\mathrm{~K} \mathrm{Gl}=$ & 0.2074821 \\
\hline 14 & U IF & 28.040032 & $\mathrm{~K} I \mathrm{IF}=$ & 0.5058555 \\
\hline 15 & $\mathrm{UFH}$ & 24.183837 & $\mathbf{K ~ F H}=$ & 0.4374084 \\
\hline 16 & $\mathrm{UIH}$ & 15.397214 & $\mathrm{~K} \mathrm{IH}=$ & 0.2797211 \\
\hline 17 & UIJ & 10.001970 & $\mathbf{K} \mid \mathrm{J}=$ & 0.1820223 \\
\hline 18 & $\mathrm{UHJ}$ & 9.202712 & $\mathbf{K ~ H J}=$ & 0.1675095 \\
\hline 19 & $\mathrm{UJJ}$ & 6.074521 & $\mathrm{~K} \mathrm{JJ}=$ & 0.1106367 \\
\hline
\end{tabular}

Figura 12: Ejemplo del diseño de un nudo típico variable para un domo.

Tabla 2. Domo Amopa de frecuencia tres.

\section{Tabla 2}


Entonces, ya que el cálculo no considera los nudos y habiendo muchas variedades de ellos, habrá que tomar en cuenta que la distancia llamada Distancia Nominal será la que arroja directamente la multiplicación de la constante K relativa a cada barra por el radio de la base sobre la cual se asienta el domo. Esta distancia será la que va del centro del nudo de un lado al centro del nudo del otro extremo.

Luego tendremos la distancia de la barra estructural del domo que dependerá exclusivamente del diseño de este, tal como se aprecia en la Figura 12.

El ejemplo mostrado pertenece al diseño de un domo el cual está realizado con barras de madera de $1 \frac{1 / 2}{2}$ × $3 "(4.00 \mathrm{~cm} \times 8.00 \mathrm{~cm})$ las cuales tienen en el extremo una platina de aluminio de $2.00 \mathrm{~cm}$ sujeta mediante dos pernos a la barra de madera, estas platinas llegan a un disco de acero de $11.00 \mathrm{~cm}$ de diámetro en el cual se fijan las platinas de las barras mediante un perno pasante. Este nudo permite la construcción sin tomar en cuenta el ángulo de llegada de cada barra lo cual lo convierte en un elemento muy eficaz para domos de menor tamaño a diferencia de los nudos rígidos donde hay que determinar el ángulo de cada barra. Los nudos rígidos, evidentemente, son más convenientes para domos que cubren grandes luces, tal es el caso del Proyecto Eden (Ver Tabla 2), claro que esto estará sujeto a un respectivo cálculo estructural.

\section{Cálculo del Domo}

Para este tipo de domos, el estudio de la frecuencia no será como en el caso de los domos poliédricos tradicionales donde se divide en tantas partes el triángulo básico ${ }^{2}$ como lo determine la frecuencia, (Ver Figura 13) en este caso nos guiaremos de un cuadrado inscrito en un círculo dentro de la proyección cuadrada de la trama cúbica (Machicao, 1990). Podemos observar en la Figura 14 como la frecuencia se determina en función a la cantidad de cuadrados de la trama que contiene por lado el cuadrado matriz pintado de color rojo, en cuyo caso tendremos frecuencia dos, tres, cuatro, etcétera.

Cabe resaltar que, a medida que aumente la frecuencia, aumentará también las posibilidades de diseño pues se incrementarán también los arcos que permiten la unión de los domos, además claro está, de obtener un domo que permitirá cubrir mayores luces, -de la misma forma que ocurre con los domos tradicionales- donde del mismo modo, al aumentar la frecuencia, se aumenta la luz al interior de estas estructuras además de dar un aspecto esférico mucho más definido (Ver Figura 15).

En la Figura $16 a y$, los estudiantes ${ }^{3}$ realizaron un modelo a escala 1:2 empleando tubos de PVC, el trabajo consistió en realizar seis módulos, uno por cada grupo, para luego unirlos según un diseño planteado por los mismos estudiantes los cuales tenían que trabajar de forma estandarizada para que luego se pudieran unir los respectivos módulos del domo Amopa.

En este trabajo académico el nudo se resolvió de forma muy sencilla calentando y aplastando los extremos del nudo para luego perforarlos y unirlos con un perno. Esta es una solución práctica y económica, ideal para trabajos académicos. 


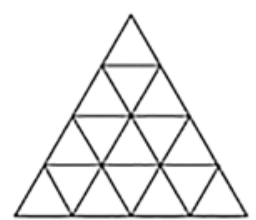

FRECUENCIA 4

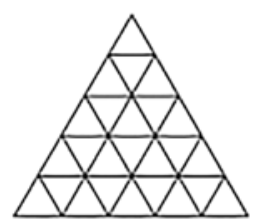

FRECUENCLA 5

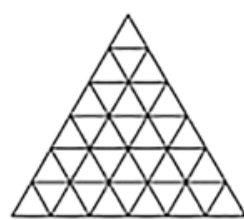

FRECUENCIA 6

\section{3}

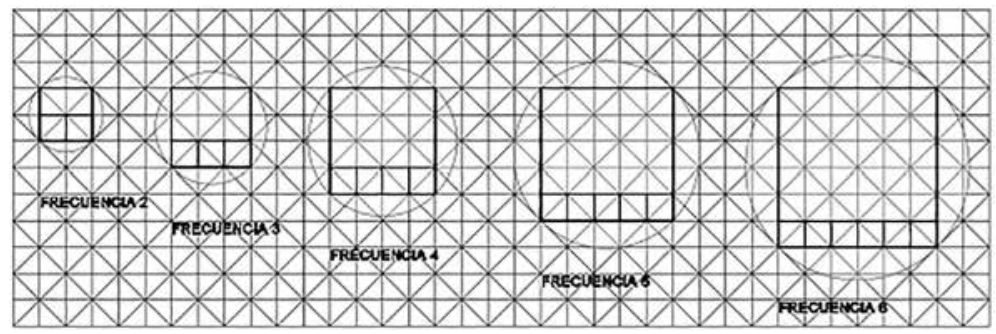

14

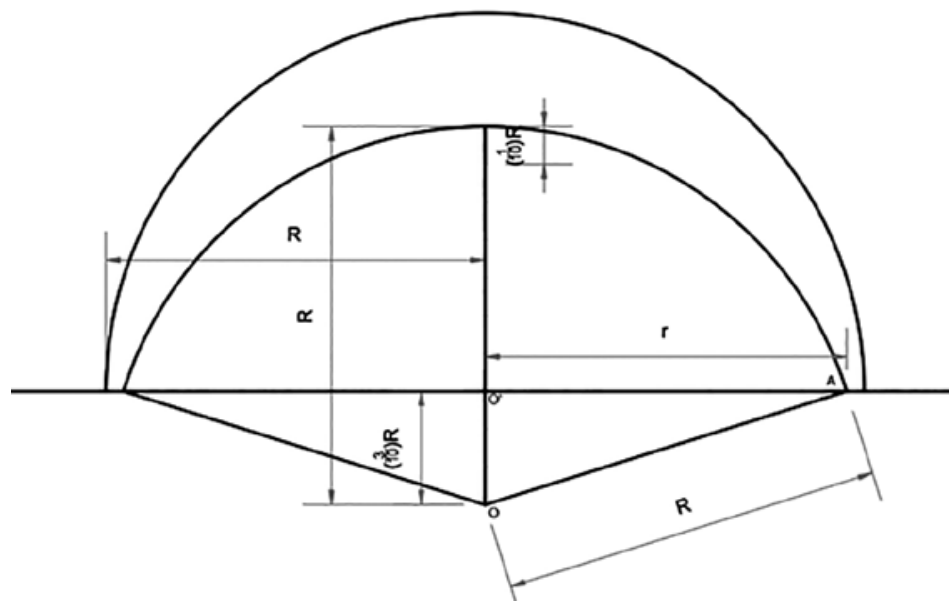

14 bis

Figura 13. Método de la frecuencia clásica de los domos tradicionales.

Figura 14. Esquema de división del rectángulo matriz para frecuencia dos, tres, cuatro, cinco y seis. Figura 15. Unión de módulos Amopa a través de arcos perpendiculares rectos al piso.

Figura 16. Trabajo compuesto por seis módulos Amopa. Desarrollado por los estudiantes del curso de Construcción III de la Carrera de Arquitectura y Urbanismo Ambiental de la Universidad Científica del Sur. Año 2018. 

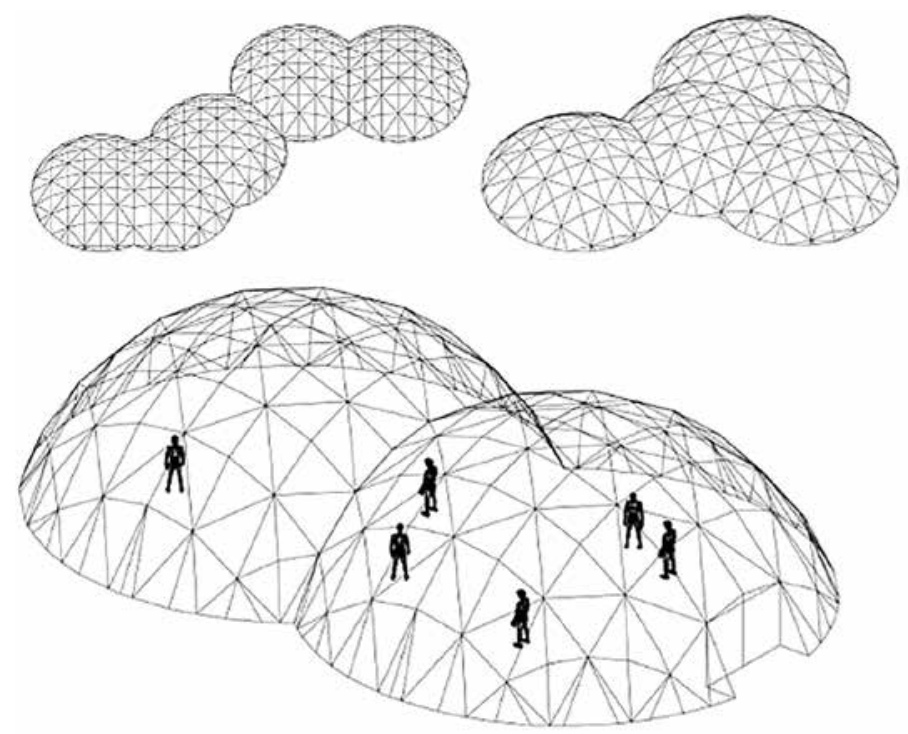

15
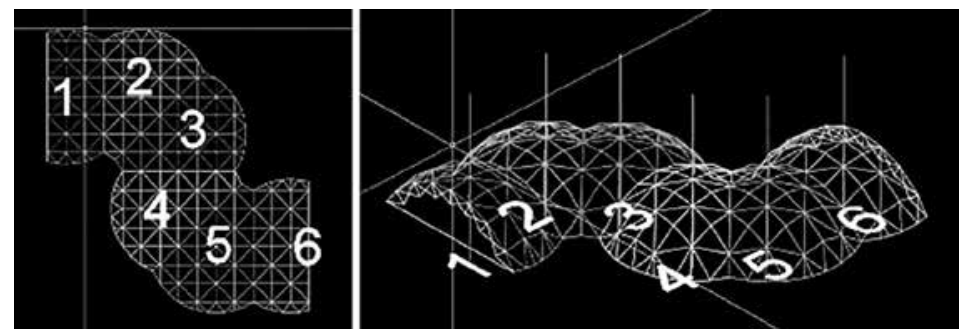

$16 \mathbf{a}$

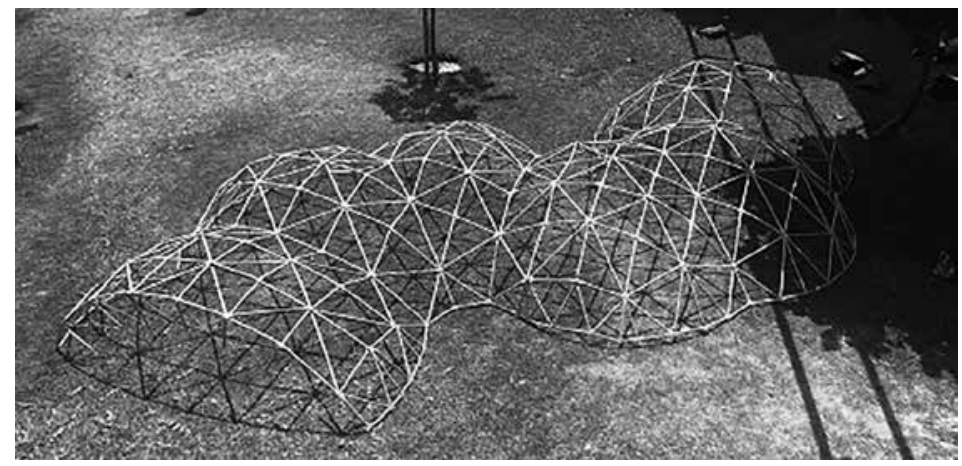

$16 b$ 
El siguiente ejemplo muestra el diseño modular de un sistema de domos que actúan como refugios en las zonas alto andinas donde estas estructuras resultan una muy buena alternativa ante las inclemencias del clima. Por otro lado, tenemos la ventaja de realizar una construcción que se amolda a las necesidades y requerimientos progresivos del usuario ya que puede ir añadiendo domos de forma progresiva.

El proyecto mostrado en Figura 17 consta de un módulo básico de vivienda con un establo adyacente el cual puede, al igual que la vivienda, crecer de forma modular tanto en sentido axial como oblicuo. La concepción de este modelo permite que los futuros trabajos de ampliación no afectan el interior del refugio hasta que, concluidas las obras de ampliación, simplemente se derrumba el muro central para conectar los ambientes nuevos con los existentes una vez que se han concluido todas las obras exteriores. En este proyecto los domos se han dispuesto de forma axial y se asientan sobre un muro tradicional de pirca ${ }^{4}$. El siguiente ejemplo de las Figuras 19 y 20 muestran parte de un proyecto integral realizado bajo el concepto de la llamada arquitectura orgánica donde se priorizan los muros y las coberturas curvas, consta de una edificación con fines de hospedaje recreacional ubicado frente a las playas de la caleta Los Órganos, Piura, Perú. Como se aprecia, la volumetría está compuesta por siete módulos debidamente acoplados unos con otros los cuales descansan sobre un muro de adobe de $0.30 \mathrm{~m}$ de espesor y $0.90 \mathrm{~m}$ de altura.

Es interesante notar que los muros que van creando los diferentes espacios arquitectónicos pueden acoplarse en perfecta armonía con la estructura de los domos, de tal suerte que la modulación se realiza en función a la trama generada por los domos Amopa. En la Figura 21 tenemos una casa huerto donde los domos anexos a los espacios destinados a la vivienda actúan como invernaderos

Como hemos visto, ya sea que proyectemos en función a la agreste geografía alto andina o para las cálidas playas tropicales del norte de Perú, existe una multitud de posibilidades morfológicas factibles gracias a la unión de estos módulos geodésicos, lo cual permitirá añadir diferentes espacios que albergarán las diversas funciones requeridas según las necesidades del usuario. 


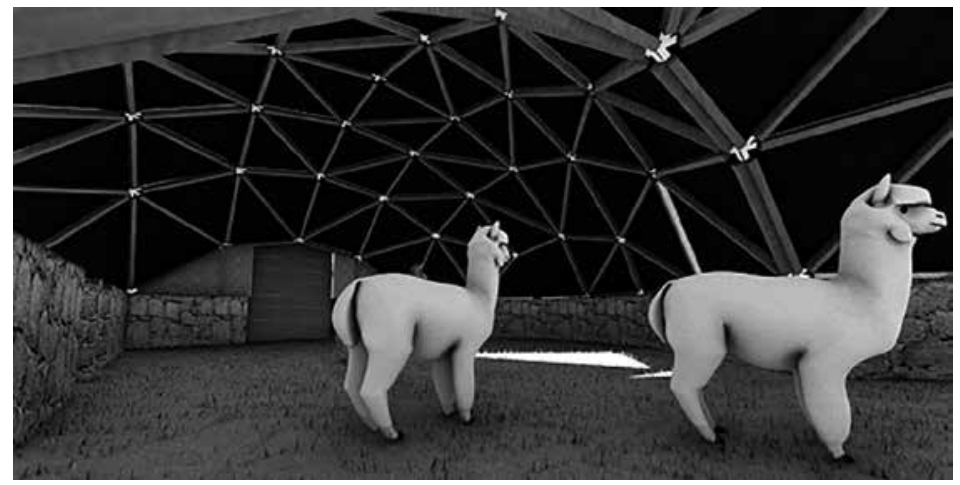

17
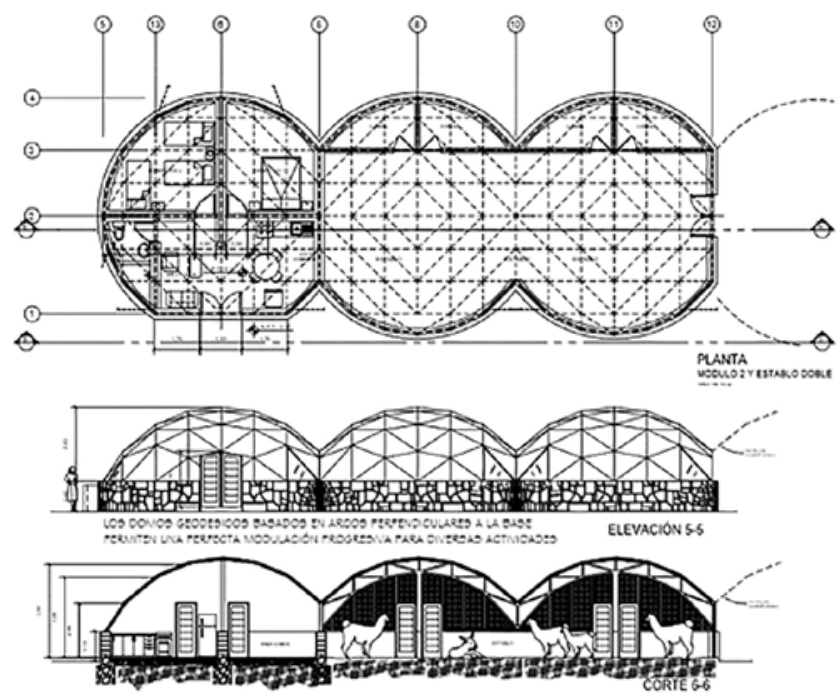

18

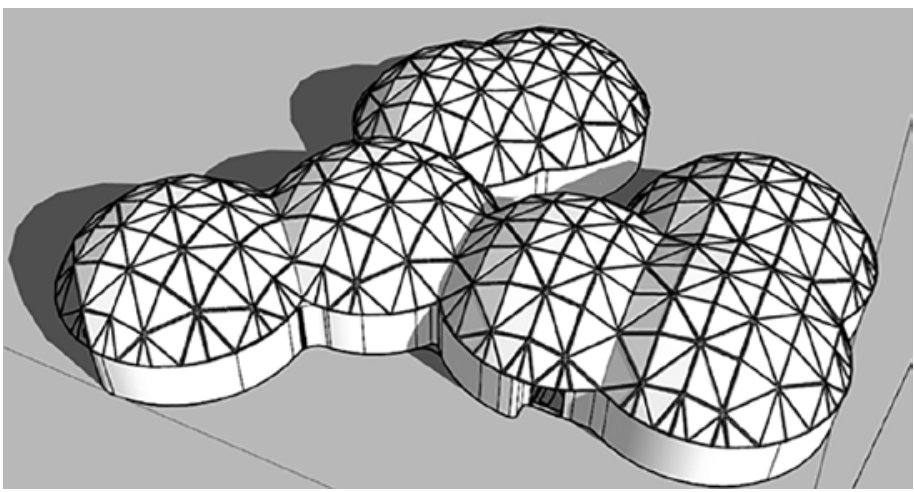

Figura 17. Interior de los domos establo (Renderización a cargo del estudiante Richard García).

Figura 18. Vivienda con establo para la región alto andina (Proyecto del Autor). Figura 19.

Vista exterior (Renderización a cargo de la estudiante Ariel Córdoba). 

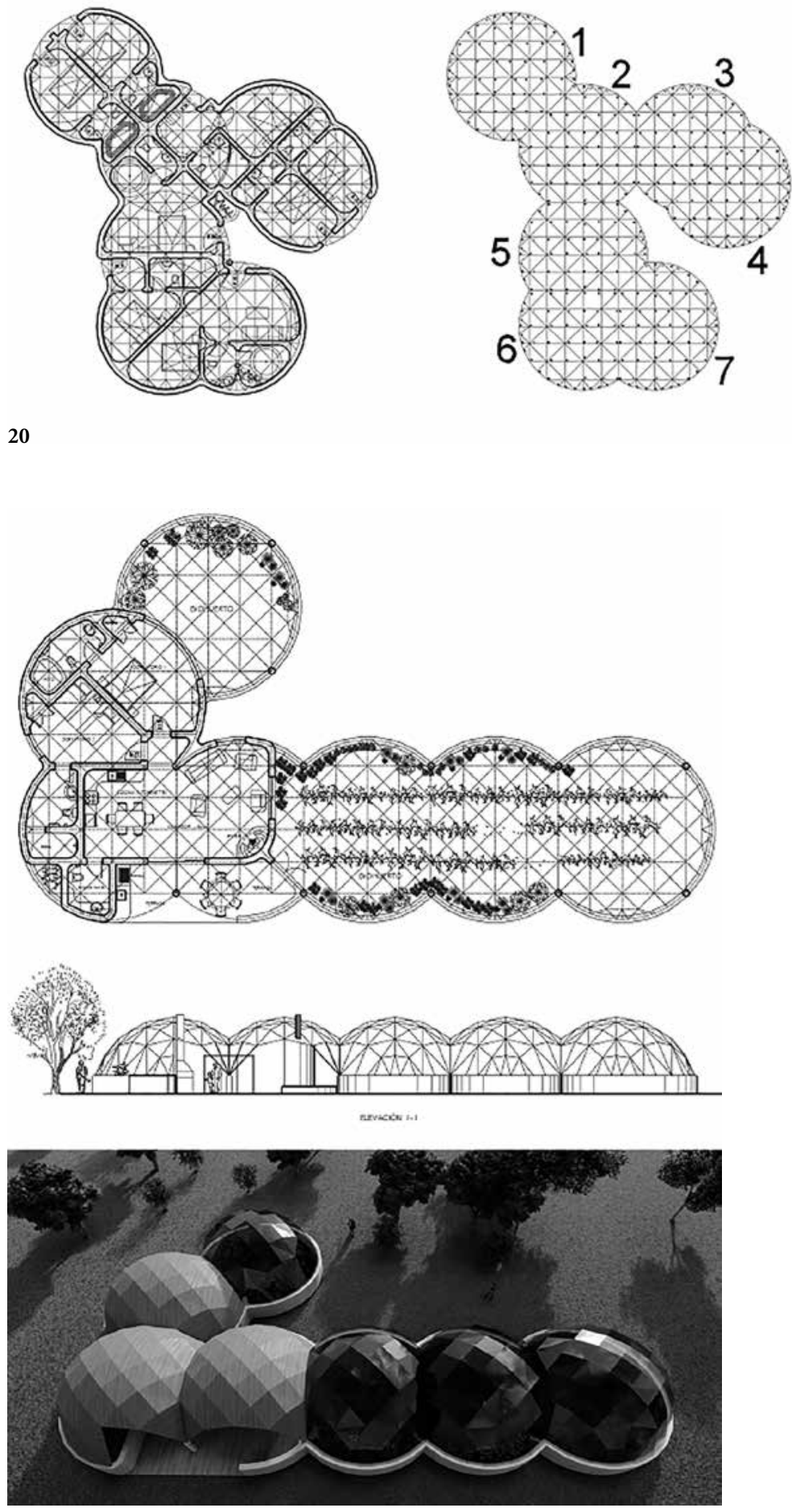

Figura 20. Trabajo compuesto por siete módulos Amopa.

(Proyecto realizado por encargo de la empresa Mundomo Eirl. Proyecto del Autor).

Figura 21. Trabajo compuesto por siete módulos Amopa. (Proyecto realizado por encargo de la empresa Warmayanay. Proyecto del Autor. Renderización a cargo del estudiante Sergio Escalante). 


\section{Constante matemática para diseñar un Domo Amopa}

Tomaremos como ejemplo un Domo Amopa de frecuencia tres el cual resulta, como ya se ha visto, de la división en tres partes del cuadrado matriz. Una vez que hemos desarrollado el domo uniendo las distintas intersecciones de los respectivos arcos, unimos el extremo inferior de la altura del centro con cada una de las intersecciones del domo. Si bien es cierto que las barras del domo se encuentran a partir de las intersecciones de los arcos -lo cual difiere de los domos poliédricos-cada punto de intersección estará ubicado de forma equidistante de un centro común ya que finalmente el domo está inscrito en una esfera. Esto nos permitirá encontrar una relación matemática entre la distancia del extremo inferior de la altura central que actuará como un radio y que será el centro común, el ángulo formado entre los vértices y los radios y, finalmente la longitud de la barra que será la estructura del domo.

Una vez realizado los diferentes modelos en CAD se pueden reproducir los domos relacionando siempre el radio de la esfera con una constante numérica la cual llamaremos constante "K", para de esta manera tener una constante relativa a cada una de las barras tal como KAB, KAC, KBC, etcétera. Esta constante relaciona -en el caso de los domos poliédricos- el ángulo entre los vértices de cada barra con el radio de la esfera del poliedro que determina el domo.

Sin embargo, el Domo Amopa se apoya con un radio menor al de la esfera que determina sus partes tal como podemos apreciar en la Figura 22 donde vemos que debido a que el centro de la esfera se ubica a 3/10 del radio bajo el plano de apoyo. Para poder emplear la fórmula que relaciona la constante $K$ con el Radio $R$ de la esfera y relacionarla con el radio $r$ de la base de apoyo debemos resolver la siguiente y sencilla operación geométrica (Ver Ecuación 1):

Ecuación $1 \quad R=\left(\frac{3}{10} \mathbf{R}\right)^{2}+r$

Resolviendo tenemos la siguiente relación:

$$
\mathrm{R}=1.044030651 \mathrm{r}
$$

La forma de obtener la constante " $\mathrm{K}$ " es similar en todos los casos donde empleamos domos poliédricos. Tomamos los siguientes datos mostrados en la Figura 22:

- Centro de radio ubicado en el extremo inferior de la altura del centro: CE

- Radio del centro CE hasta uno de los vértices de las barras: $\mathbf{R}$

- Angulo entre radios: $\Phi$

- Barra del domo: AB 


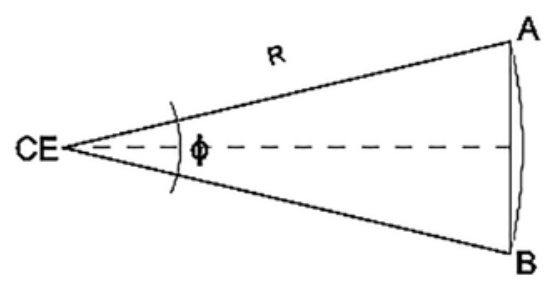

22

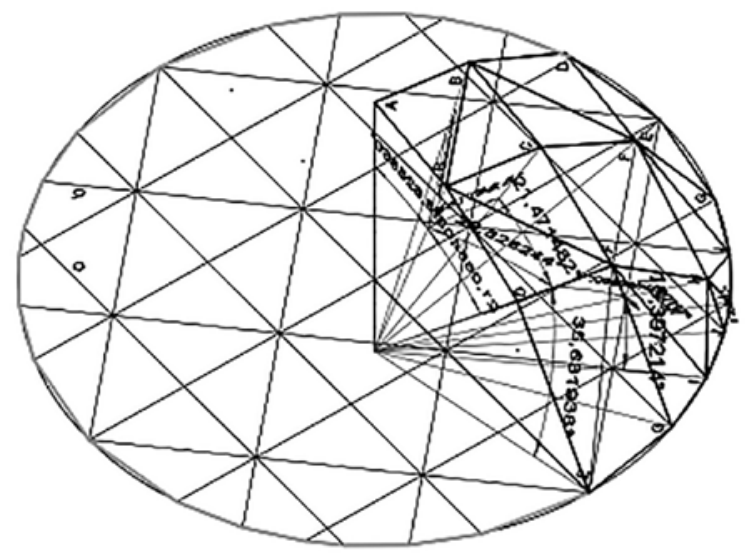

23

Tenemos por trigonometría elemental:

$$
\begin{aligned}
& \text { Sen } \Phi / 2=(A B / 2) / R \\
& A B=2 . R . \operatorname{Sen} \Phi / 2 \\
& \text { Hacemos } K=2 \text { Sen } \Phi / 2
\end{aligned}
$$

De modo que tenemos la siguiente fórmula:

$$
\mathrm{AB}=\mathrm{R} \mathrm{KAB}
$$

Donde $R$ es el radio de la esfera que contiene el domo, pero como el domo se asienta sobre un círculo de radio $r$ reemplazamos el radio $R$ y obtenemos la siguiente expresión:

$$
\mathrm{AB}=1.044030651 \mathrm{r} \mathrm{KAB}
$$

De esta manera diseñaremos los domos Amopa considerando el radio $r$ de apoyo. 


\section{Conclusiones}

Ya sea que proyectemos en función a la agreste geografía alto andina o para las cálidas playas tropicales del norte de Perú, existe una multitud de posibilidades morfológicas factibles gracias a la unión de módulos geodésicos, esto permitirá añadir diferentes espacios que albergarán las diversas funciones requeridas según el requerimiento del usuario.

La única restricción es que estos Domos no generan media esfera lo cual requiere de un zócalo sobre el cual descansa la estructura. Esto, en realidad más que restricción podría considerarse una característica pues es siempre una ventaja contar con un elemento que aísle y proteja el domo del piso.

El mundo del diseño es vasto y fascinante donde la única limitación es nuestra propia imaginación.

\begin{tabular}{|c|c|c|c|c|}
\hline & AMOPA F4 & \begin{tabular}{|l|} 
Diedro \\
\end{tabular} & & \\
\hline & \multicolumn{2}{|c|}{ BARRAS DE CRUCERIA } & & \\
\hline 1 & U AB & 13.854468 & $\mathrm{KAB}=$ & 0.24121741 \\
\hline 2 & $\mathrm{UAD}$ & 19.794203 & $\mathrm{KAD}=$ & 0.34375853 \\
\hline 3 & $\mathrm{UBD}$ & 13.860823 & $\mathrm{~K} \mathrm{BD}=$ & 0.24132752 \\
\hline 4 & U BC & 14.759974 & $\mathrm{~K} \mathrm{BC}=$ & 0.25689841 \\
\hline 5 & U CD & 19.835160 & $\mathrm{KCD}=$ & 0.3444627 \\
\hline 6 & U CF & 13.885756 & $\mathrm{KCF}=$ & 0.24175949 \\
\hline 7 & U DI & 22.837678 & $\mathrm{~K} \mathrm{DI}=$ & 0.39595929 \\
\hline 8 & U FI & 15.114676 & $\mathrm{~K} \mathrm{Fl}=$ & 0.2630366 \\
\hline 9 & U CE & 17.305602 & $\mathrm{KCE}=$ & 0.30089294 \\
\hline 10 & U EH & 13.968953 & $\mathrm{KEH}=$ & 0.24320084 \\
\hline 11 & $\mathrm{UH}$ & 20.073741 & $\mathrm{~K} \mathrm{HI}=$ & 0.34856375 \\
\hline 12 & U EG & 27.380710 & $K E G=$ & 0.47334919 \\
\hline 13 & $\mathrm{UGH}$ & 28.865567 & $\mathrm{~K} \mathrm{GH}=$ & 0.4984881 \\
\hline 14 & $\mathrm{UFH}$ & 17.634379 & $\mathrm{~K} \mathrm{FH}=$ & 0.30656462 \\
\hline 15 & UFD & 14.830037 & $K \mathrm{FD}=$ & 0.25811106 \\
\hline
\end{tabular}

\begin{tabular}{|c|c|c|c|c|}
\hline & \multicolumn{2}{|c|}{ BARRAS DE CONTRAFUERTE } & & \\
\hline 16 & UGJ & 13.863873 & K GJ= & 0.24138036 \\
\hline 17 & U JH & 24.322021 & $\mathrm{~K} \mathrm{JH}=$ & 0.42131924 \\
\hline 18 & U JK & 9.402673 & $\mathrm{~K} \mathrm{JK}=$ & 0.16392351 \\
\hline 19 & U HK & 24.607165 & $\mathrm{~K} \mathrm{HK}=$ & 0.42618295 \\
\hline 20 & $\mathrm{UHL}$ & 16.200418 & $\mathrm{KHL}=$ & 0.28180969 \\
\hline 21 & U LK & 16.084290 & $\mathrm{~K} L \mathrm{LK}=$ & 0.27980295 \\
\hline 22 & U LI & 19.158526 & $\mathrm{~K} \mathrm{LI}=$ & 0.33282375 \\
\hline 23 & U IM & 15.334435 & $\mathrm{~K} \mid \mathrm{M}=$ & 0.26683832 \\
\hline 24 & U LM & 9.826069 & $K$ LM= & 0.17128717 \\
\hline 25 & $\mathrm{U} \mathrm{KN}$ & 5.464035 & $\mathrm{~K} \mathrm{KN}=$ & 0.09532927 \\
\hline 26 & U LN & 14.014871 & $\mathrm{~K} L N=$ & 0.2439963 \\
\hline 27 & UNO & 10.926937 & $\mathrm{~K} \mathrm{NO}=$ & 0.19042215 \\
\hline 28 & U LO & 15.322666 & $K \mathrm{LOO}=$ & 0.26663475 \\
\hline 29 & U OM & 15.676019 & $\mathrm{~K} \mathrm{OM}=$ & 0.27274559 \\
\hline 30 & U OP & 3.438912 & $\mathrm{KOP}=$ & 0.06001133 \\
\hline 31 & U MP & 15.334440 & K MP= & 0.26683841 \\
\hline
\end{tabular}

\section{Tablas anexas}

Tabla 3. Domo Amopa de frecuencia cuatro. 


\begin{tabular}{|c|c|c|c|c|}
\hline & AMOPA F5 & Diedro & & \\
\hline & \multicolumn{2}{|c|}{ BARRAS DE CRUCERIA } & & \\
\hline 1 & U AB & 11.044145 & $\mathrm{KAB}=$ & 0.19245842 \\
\hline 2 & U BB & 15.570104 & $\mathrm{KBB}=$ & 0.27091418 \\
\hline 3 & U BD & 11.483584 & $\mathrm{KBD}=$ & 0.20009105 \\
\hline 4 & $\mathrm{UBF}$ & 16.204656 & $\mathrm{KBF}=$ & 0.28188292 \\
\hline 5 & $\mathrm{UBC}$ & 11.046137 & $\mathrm{KBC}=$ & 0.19249302 \\
\hline 6 & UDF & 11.053162 & $\mathrm{KDF}=$ & 0.19261506 \\
\hline 7 & $\mathrm{UCF}$ & 11.503838 & $\mathrm{KCF}=$ & 0.20044277 \\
\hline 8 & UFF & 15.570104 & K FF= & 0.27091418 \\
\hline 9 & UDE & 12.550600 & $\mathrm{KDE}=$ & 0.21861162 \\
\hline 10 & UEF & 16.327708 & $\mathrm{KEF}=$ & 0.28400898 \\
\hline 11 & UEG & 14.940980 & $K E G=$ & 0.26003107 \\
\hline 12 & U GK & 11.120288 & $\mathrm{~K}$ GK= & 0.19378116 \\
\hline 13 & U EK & 19.562271 & $K$ EK= & 0.33977009 \\
\hline 14 & $\mathrm{UEH}$ & 11.070131 & $\mathrm{~K} \mathrm{EH}=$ & 0.19290985 \\
\hline 15 & $\mathrm{UHL}$ & 11.755991 & $\mathbf{K ~ H L}=$ & 0.20482103 \\
\hline 16 & $\mathrm{UFL}$ & 18.734276 & $\mathrm{KFL}=$ & 0.32552017 \\
\hline 17 & $\mathrm{UFI}$ & 11.576066 & $\mathrm{~K} \mathrm{FI}=$ & 0.201697 \\
\hline 18 & UIL & 12.898686 & $\mathrm{~K}$ IL= & 0.22464944 \\
\hline 19 & UG & 23.281446 & $\mathrm{KGJ}=$ & 0.40354821 \\
\hline 20 & U JK & 24.470076 & $\mathrm{~K} \mathrm{JK}=$ & 0.42384495 \\
\hline 21 & $\mathrm{UKL}$ & 16.809631 & $\mathrm{~K} \mathrm{KL}=$ & 0.29233235 \\
\hline 22 & $\mathrm{UFH}$ & 12.625022 & $\mathrm{~K} \mathrm{FH}=$ & 0.2199027 \\
\hline 23 & U HK & 15.196899 & $\mathbf{K ~ H K =}$ & 0.26445913 \\
\hline 24 & U LL & 15.570104 & $\mathbf{K ~ L L}=$ & 0.27091418 \\
\hline
\end{tabular}

\begin{tabular}{|c|c|c|c|c|}
\hline & AMOPA F6 & Diedro & & \\
\hline & \multicolumn{2}{|c|}{ BARRAS DE CRUCERIA } & & \\
\hline 1 & UAB & 9.185863 & $\mathrm{KAB}=$ & 0.16015191 \\
\hline 2 & UAD & 13.047680 & $\mathrm{KAD}=$ & 0.22723323 \\
\hline 3 & UBC & 9.433241 & $\mathrm{KBC}=$ & 0.16445522 \\
\hline 4 & $U \mathrm{BD}$ & 9.186636 & $\mathrm{KBD}=$ & 0.16016535 \\
\hline 5 & $U \mathrm{UCD}$ & 13.052317 & $\mathrm{KCD}=$ & 0.22731364 \\
\hline 6 & UCF & 9.189269 & $\mathrm{KCF}=$ & 0.16021116 \\
\hline 7 & UDF & 9.440861 & $K D F=$ & 0.16458777 \\
\hline 8 & UDI & 13.793802 & $\mathrm{KDI}=$ & 0.24016629 \\
\hline 9 & UFI & 9.466524 & $\mathrm{~K} \mathrm{DI}=$ & 0.16503415 \\
\hline 10 & UEC & 9.995332 & $\mathrm{KEC}=$ & 0.17423032 \\
\hline 11 & UEH & 9.194796 & $\mathrm{KEH}=$ & 0.16030731 \\
\hline 12 & $\mathrm{UCH}$ & 13.843953 & $\mathrm{~K} \mathrm{CH}=$ & 0.24103523 \\
\hline 13 & UFH & 10.020711 & $\mathrm{KFH}=$ & 0.17467158 \\
\hline 14 & UHI & 13.069993 & $\mathrm{KHI}=$ & 0.22762014 \\
\hline 15 & UEG & 11.069407 & $K E G=$ & 0.19289727 \\
\hline 16 & U GH & 14.038953 & $\mathrm{~K} \mathrm{GH}=$ & 0.24441346 \\
\hline 17 & U GK & 9.206616 & K GK= & 0.16051295 \\
\hline 18 & U HK & 11.137788 & $\mathrm{KHK}=$ & 0.19408515 \\
\hline 19 & $U G$ & 13.273623 & $K G J=$ & 0.2311507 \\
\hline 20 & U GN & 16.920349 & $\mathrm{KGN}=$ & 0.29424385 \\
\hline 21 & U KN & 13.475139 & $\mathrm{~K} \mathrm{KN}=$ & 0.23464389 \\
\hline 22 & U JN & 9.239437 & $\mathrm{KJN}=$ & 0.16108393 \\
\hline 23 & U JM & 20.343285 & $\mathrm{~K} J \mathrm{JM}=$ & 0.35319522 \\
\hline 24 & UMN & 21.339911 & $\mathrm{KMN}=$ & 0.37030266 \\
\hline 25 & UNN & 14.671695 & $\mathrm{~K} \mathrm{NN}=$ & 0.25537034 \\
\hline 26 & U KN & 9.641924 & $\mathrm{~K} \mathrm{KN}=$ & 0.16808482 \\
\hline 27 & U HN & 16.006306 & $\mathrm{~K} \mathrm{HN}=$ & 0.27845519 \\
\hline 28 & U NLL & 11.376369 & $K N L=$ & 0.1982291 \\
\hline 29 & UHL & 9.521490 & $\mathrm{~K} \mathrm{HL}=$ & 0.1659902 \\
\hline 30 & UÑO & 13.122670 & K NO= & 0.22853353 \\
\hline 31 & $\mathrm{UOL}$ & 10.297088 & $\mathrm{KOL}=$ & 0.17947633 \\
\hline 32 & U10 & 15.790400 & $\mathrm{~K} \mid \mathrm{O}=$ & 0.27472313 \\
\hline 33 & U IL & 10.106994 & $\mathrm{~K} \mid \mathrm{L}=$ & 0.1761717 \\
\hline
\end{tabular}

\begin{tabular}{|r|r|r|l|r|}
\hline & BARRAS DE CONTRAFUERTE & & \\
\hline 25 & U JM & 11.048855 & $\mathrm{~K} \mathrm{JM}=$ & 0.19254024 \\
\hline 26 & U KM & 21.243927 & $\mathrm{~K} \mathrm{KM}=$ & 0.36865626 \\
\hline 27 & U MN & 8.005835 & $\mathrm{~K} \mathrm{MN}=$ & 0.13961454 \\
\hline 28 & U KN & 21.749470 & $\mathrm{~K} \mathrm{KN}=$ & 0.37732485 \\
\hline 29 & U KP & 12.339635 & $\mathrm{~K} \mathrm{KP}=$ & 0.21495128 \\
\hline 30 & U NO & 3.526924 & $\mathrm{~K} \mathrm{NO}=$ & 0.06154672 \\
\hline 31 & U PN & 15.583836 & $\mathrm{~K} \mathrm{PN}=$ & 0.27115164 \\
\hline 32 & U LP & 16.228066 & $\mathrm{~K} \mathrm{LP}=$ & 0.28228741 \\
\hline 33 & U PO & 14.607493 & $\mathrm{~K} \mathrm{PO}=$ & 0.25425893 \\
\hline 34 & U OQ & 12.728789 & $\mathrm{~K} \mathrm{OQ}=$ & 0.2217027 \\
\hline 35 & U PQ & 17.146319 & $\mathrm{~K} \mathrm{PQ}=$ & 0.29814428 \\
\hline 36 & U LQ & 29.733224 & $\mathrm{~K} \mathrm{LQ}=$ & 0.51313923 \\
\hline 37 & U LR & 13.644413 & $\mathrm{~K} \mathrm{LR}=$ & 0.23757762 \\
\hline 38 & U RQ & 20.272113 & $\mathrm{~K} \mathrm{RQ}=$ & 0.35197249 \\
\hline 39 & U QQ & 15.570104 & $\mathrm{~K} \mathrm{QQ}=$ & 0.27091418 \\
\hline
\end{tabular}

Tabla 4 (arriba). Domo Amopa de frecuencia cinco.

Tabla 5 (derecha). Domo Amopa de frecuencia seis.

\begin{tabular}{|c|c|c|c|c|}
\hline \multicolumn{5}{|c|}{ BARRAS DE CONTRAFUERTE } \\
\hline 34 & UMP & 9.188562 & $K X X=$ & 0.16019886 \\
\hline 35 & UNP & 18.874581 & $K N P=$ & 0.32793606 \\
\hline 36 & UPQ & 6.973019 & $K P Q=$ & 0.12162705 \\
\hline 37 & UNQ & 19.454821 & $\mathrm{KNQ}=$ & 0.33792185 \\
\hline 38 & UNR & 10.000646 & K NR= & 0.17432272 \\
\hline 39 & UNR & 14.228185 & KNR= & 0.24769109 \\
\hline 40 & URQ & 14.722905 & $K R Q=$ & 0.25625678 \\
\hline 41 & UQT & 2.487172 & $K Q T=$ & 0.04340593 \\
\hline 42 & URU & 14.426764 & $K R U=$ & 0.2511299 \\
\hline 43 & URT & 14.180728 & K RT $=$ & 0.24686917 \\
\hline 44 & $U T U$ & 5.801866 & $\mathrm{KTU}=$ & 0.10121841 \\
\hline 45 & URV & 12.396265 & $K R V=$ & 0.2159339 \\
\hline 46 & $U \overline{N V}$ & 23.296235 & $K N V=$ & 0.40380101 \\
\hline 47 & UVU & 6.984397 & $K V U=$ & 0.12182526 \\
\hline 48 & UUX & 4.282563 & $K \cup X=$ & 0.07472743 \\
\hline 49 & $U V X$ & 4.821580 & $K \vee X=$ & 0.08412762 \\
\hline 50 & $U X Y$ & 1.309216 & $K X Y=$ & 0.02284963 \\
\hline 51 & $U V Z$ & 5.331358 & $\mathrm{~K} \mathrm{VZ}=$ & 0.09301619 \\
\hline 52 & UVY & 4.750151 & $\mathrm{~K} \vee \mathrm{Y}=$ & 0.08288203 \\
\hline 53 & UNS & 10.742834 & KNS= & 0.1872233 \\
\hline 54 & USV & 16.427396 & $\mathrm{KSV}=$ & 0.28573112 \\
\hline 55 & UOS & 11.938022 & $\mathrm{KOS}=$ & 0.2079811 \\
\hline 56 & U SW & 13.513060 & K SW $=$ & 0.23530115 \\
\hline 57 & U OW & 21.929674 & $\mathrm{KOW}=$ & 0.38041305 \\
\hline 58 & U VW & 13.477692 & $\mathrm{~K} \mathrm{WW}=$ & 0.23468814 \\
\hline 59 & UWZ & 13.784636 & K WZ= & 0.24000747 \\
\hline 60 & UYZ & 2.098056 & K WZ $=$ & 0.03661594 \\
\hline 61 & $\cup Z 3$ & 7.904380 & $K Z Z=$ & 0.13784808 \\
\hline 62 & UWB & 9.230769 & $K W B=$ & 0.16093313 \\
\hline 63 & $U ß \varphi$ & 3.055131 & $\mathrm{~K} B \varphi=$ & 0.05331578 \\
\hline 64 & $U W_{\varphi}$ & 8.739200 & $K W \varphi=$ & 0.15238 \\
\hline
\end{tabular}




\title{
Notas
}

1. El término frecuencia va referido la división que se realiza en las caras del poliedro que se está empleando a fin de darle mayor resistencia al domo y mejorar la forma exterior de tal suerte que, al aumentar la frecuencia, la curvatura del domo tenderá a convertirse en la superficie de una esfera. N. del A.

2. En los casos de domos generados por un hexaedro se hablará de cuadrados esféricos y en estos cuadrados se dividirán las aristas en tantas partes como lo determine la frecuencia. De forma similar ocurrirá en el caso del domo generado a partir de un dodecaedro donde la frecuencia se verá en cada uno de los cinco triángulos que forman el pentágono esférico. Esto se analiza en el libro "De Cúpulas Geodésicas, Fractales, Tenségritys y Algo Más" escrito por el autor.

3. Estudiantes del curso de Construcción III de la Carrera de Arquitectura y Urbanismo Ambiental de la Universidad Científica del Sur. Semestre 2018-1.

4. El término "pirca" proviene del vocablo quechua pirqa, el cual se refiere a una pared, un muro o una valla usualmente de piedra sin cantear, estos suelen ser muros de construcción rústica y baja altura, los cuales están realizados con piedras sin labrar calzadas sin el uso de mortero, este método es ampliamente utilizado por los pueblos alto andinos.

\section{Referencias}

Bassegoda, J. (1985). Gaudi. Barcelona: Salvat Editores S.A.

Machicao, R. (1990). Diseño Estructural Para Arquitectos. Lima: Editorial Arius S.A.

Martínez Cendra, F. (2014). De cúpulas geodésicas, fractales, tensegritys y algo más. Universidad de San Martín de Porres. Fondo Editorial.

Otto, F. (1973). Frei Otto: Estructuras. Barcelona: Editorial Gustavo Gilli.

Rice, P. (2016). Un Ingeniero Imagina. Madrid: CINTER Divulgación Técnica.

\begin{abstract}
Geodesic domes have been designed in various places, mainly based on polyhedral figures such as the Icosahedron. This supposes buildings with a circular plan that generally must act independently since the geometry of each element does not allow the union of domes unless the basic geometry is forced to achieve coupling these buildings as soap bubbles, where in which case various elements will have to be trimmed in order to achieve this union.

This represents a structural problem that must be solved by means of elements other than the basic geometry of the dome and that somehow solve the trauma of having sectioned the structure into different parts.

The objective of this article is to study an alternative design form that allows the union of domes without altering its own basic geometry.
\end{abstract}


Keywords: Architecture - Union of domes - Interconnected geodesic domes - Portuguese

Resumo: Cúpulas geodésicas foram projetadas em vários lugares, começando principalmente com figuras poliédricas como o Icosaedro. Isso supõe edifícios com uma planta circular que geralmente devem atuar de forma independente uma vez que a geometria de cada elemento não permite a união de cúpulas a menos que a geometria básica seja forçada a conseguir o acoplamento desses edifícios como bolhas de sabão, onde neste caso vários elementos deverão ser corte para conseguir essa união.

Isso representa um problema estrutural que deve ser resolvido por meio de outros elementos além da geometria básica da cúpula e que de alguma forma resolvem o trauma de ter seccionado a estrutura em partes diferentes.

O objetivo deste artigo é estudar uma forma de projeto alternativa que permite a união de cúpulas sem alterar sua própria geometria básica.

Palavras chave: Arquitetura orgânica - União de cúpulas - Cúpulas geodésicas interconectadas 